\title{
EL GERENTE DESCARTABLE: UN DILEMA ENTRE LA OPTIMIZACIÓN DE LA PRODUCTIVIDAD Y LA MINIMIZACIÓN DEL BIEN-ESTAR SOCIAL.
}

\section{Istvan Karoly Kasznar}

\section{RESUMEN}

Este artículo caracteriza y muestra una importante alternativa de contratación de trabajadores especializados, de buena formación superior, normalmente universitária, que se ven transformados en vendedores, hazen parte de la fuerza de ventas y promueven en La realidad los resultados económicos y financieros empresariales que se buscan en políticas de Marketing. El autor muestra que motivadas por la busca de resultados inmediatos, numerosas instituciones financieras han optado por la contratación de una mano de obra que es predominantemente descartable. Se usa, se pone fuera. Enquanto esa mano de obra tiene y muestra alta productividad y el mercado de crédito está caliente, el trabajador se mantiene en la empresa. Basta que los rendimientos, que son naturalmente cíclicos, caigan, que la amenaza de la demision y del descarte ocurra. Esto crea sequelas en las empresas, entre ellas la falta de fidelidad. Y en los sujetos descartados, puede producir efectos como desmotivación, depresión, y falta de cooperativismo con la empresa originalmente contratante.

\section{RESUMO}

Este artigo caracteriza e mostra uma alternativa importante de contratação de trabalhadores especializados, de boa formação superior, universitária normalmente, que se transformam em vendedores, fazem parte da força de vendas e promovem na realidade o que se busca de resultados em políticas de Marketing. $\mathrm{O}$ autor mostra que movidas pela busca imediata de resultados, numerosas instituições financeiras optaram pela contratação de uma mão de obra essencialmente descartável. Enquanto ela possui produtividade e o mercado de crédito está aquecido, ela é mantida e paga com um salário acima da média de mercado. Basta os rendimentos não serem tão bons, baixar a produtividade média do trabalhador, ou ser o mercado de crédito cadente por conta de uma recessão, que esse profissional é descartado, logo, demitido. Isto possui seqüelas empresariais, entre elas a falta de fidelidade. E nos trabalhadores descartados, pode produzir efeitos como desmotivação, depressão e falta de cooperativismo com a empresa originalmente contratante. 


\section{APRESENTACIÓN}

Suele ser bastante comun en los dias que corren el acto de contratar profesionales em el nivel de gerencia por un periodo relativamente corto de tiempo, durante el qual lãs empresas presionan los indivíduos a dar el máximo rendimiento, mediante la entrega de um premio, un bonus o un salário mayor en contrapartida, bajo la presión de demitir, caso no se confirme a corto plazo ese rendimiento.

Por un lado, los indivíduos buscan la seguridad, la permanencia, la Carrera asciendiente y la alta remuneración en un buen empleo, ofrecido por empresas de porte que sean líderes de mercado. Por otro lado, las empresas modernas, movidas por competición, competitividad y presiones del Consejo de Administración y de los proprietários, visan El lucro alto, dividendos recordes y la capacitación para investir más, lo que requiere ahorros altos.

De esta forma, mismo que trabajadores y empresas busquen juntos la producción y la productividad máximas, en lo que son aliados, surge el antagonismo entre ellos, puesto que el resultado empresarial tiene que ser dividido entre capitalistas y trabajadores.

Para disminuir el costo de la mano de obra, las empresas usan diversos expedientes. Entre ellos, el de mantener por tiempo limitado los gerentes y la fuerza de ventas. Al menor señal de reducción de su productividad financiera, son demitidos, sin piedad y sin que se evaluen las consequencias sociales de ese acto unilateral.

La figura del gerente descartable aparece en este ambiente. Si no se adapta, o no ES adaptable a las circunstancias del momento económico, es demitido. No más. Esto tiene profundas consequencias en las empresas, que pueden ganar o perder mucho, en los aspectos financiero, productivo, de motivación, y ético, entre otros, en este contexto. El personaje del gerente descartable es sobretodo relacionado a ventas y a políticas de marketing. Si vende y promueve las ventas, el descartable se queda, es mantenido en La empresa. Caso contrário, ya sabe que es bien posible que sea demitido. En el mundo del marketing, lo que se desea es hacer mercado, conquistar mercado y más resultados. Luego, hay que saber vender y crecer sobre los concorrentes.

La habilidad de ventas no pertenece a todas las personas. Es algo de muy único, um bien raro y precioso, que depende de talento, de extroversión, de contactos, de relaciones, de capacidad de comunicación, de simpatia, de empatia y factores determinantes Del genero.

Entonces, descobrir en la fuerza de ventas quien es hábil vendedor, y quien no lo ES - puede tener otras competencias - corresponde a un gran desafio de las empresas. Afinal de cuentas, todas quieren vender y necesitan hacerlo. Entonces, pagan bien a quien sabe ahacerlo y no valorizan tanto a los que actuan en el back-office.

Este estudio adentra en este asunto, para entender el significado, la importancia y lãs consequencias principales del uso y de la contratación de los así denominados gerentes descartables.

\section{ANTECEDENTES}

En la Teoria Microeconomica, que estudia los precios de mercado; el consumidor; los productores; la distribución de ingresos y efectos entre variables económicas, se encuentra el epicentro de la mentalidad clásica extractiva en Economia, que dice: "para maximizar lucros, 
es necesário maximizar las ventas y minimizar los costos".

Esta frase es muy poderosa, está profundamente arraigada en las mentes liberales y aunque invite el analista a un acuerdo prévio de primera instancia, no pasa ni puede ser aprovada en una segunda y más meticulosa lectura. De hecho, hace sentido vender mucho, si hay demanda y el precio es atraente para dar retornos reales y dividendos apetitosos a los accionistas de una empresa. Y también es de buen alvitre controlar los costos y verlos bajos.

Contodo, los clásicos se olvidan de que las ventas maximizadas pueden llevar a producciones abusivas, en las quales se pone en riesgo la calidad del producto. Habrá gente y máquinas y equipamientos suficientemente bien calificados, para hacer correctamente La entrega de los productos? De la misma forma, es fácil cortar costos, como disminuir salários; demitir gente senior al señal de encorpamiento de beneficios y derechos; comprar matérias primas de segunda calidad; o embalar menos producto en el mismo envase.

Pero lo que realmente cuenta es la manutención de la calidad, del cliente y de su fidelidad para con productos y servicios, lo que implica en serenamente equilibrar lãs necesidades de obtención de lucros económico-financieros, con las exigencias de calidad y capacidad en poner productos de calidad perenemente en las manos del consumidor. El asunto adquiere complexidad, en la medida en que los accionistas exigen máximos resultados en dividendos; máximos resultados en nuevas inversiones en El negócio; y mínimo endeudamiento, para no caer en el pago de interés, que hace con que los lucros caigan o desaparezcan.

Haya vista este quadro, independiente del calibraje que requiera la correcta administración de negócios, es común que la administración directiva de las empresas use la visión del "quanto más, en menos tiempo, tanto mejor", a las expensas de su fuerza de ventas y luego de ciertos perfiles de gerencia. Es en este quadro que entra y aparece el gerente descartable.

Que es este gerente, dicho descartable? Corresponde a aquel profesional que ES contratado por las empresas para dar un producto o servicio bien especificado, por uma remuneración atraente, y cuya productividad tiene que ser bien superior a la média.

Enquanto rinda, el gerente se mantendrá. Contodo, caso repita dos o más vezes bajos o malos rendimientos, independiente frequentemente de los aires y cenários macroeconómicos, será demitido, defenestrado impiedosamente y sin apelos de ninguna espécie.

Pragmática, la empresa pondrá alguien inmediatamente en el lugar del gerente descartado, tal y qual una lamina de barbear, que una vez usada, se tira a la basura sin sentimientos. Y de nuevo buscará en el recién contratado la maximización de las ventas y de los resultados.

El gerente descartado se verá en la calle, a la busca de un empleo. Amargurará um desempleo por incompetencia, incapacidad o hasta injusticia, puesto que puede ser muy capaz, pero el ambientre de trabajo no le dió las oportunidades de mostrar su verdadero desempeño potencial y efectivo.

La práctica muestra que este expediente es muy comun en el mundo empresarial. Bancos, empresas de telecomunicaciones, revendedoras y distribuidoras de productos a granel, al pormenor, tienen el gusto de contratar gerentes, darles un entrenamiento básico, ponerles metas de productividad elevada y exigir resultados cada vez más inmediatos.

Caso los resultados sean alcanzados, los gerentes descartables podrán, segun el tipo de contrato que hayan hecho con las empresas, mantener-se por más un periodo (trimestre; 
semestre o año) como empleados; ganar o no bonus y premios; recibir acciones; subir en La jerarquia; y promoverse en un plano de carreras.

Contodo, caso no atinjan los resultados pretendidos, independiente de los factores que explican su fracaso, serán demitidos y perderán su empleo, sin rendimientos especiales adicionales. Puesto que son el elo flaco de esta relación, les caberá el desempleo, El opróbio, el olvido, la dificultad de encontrar empleo nuevo, en un mundo en el qual tanto jóvenes quanto más experientes se ven a vueltas con el espectro de la ociosidad y de salários volátiles y cadentes.

Se estima que $78 \%$ de la mano de obra especializada de buen nivel que actua en El sector de servicios bancários en el Brasil, en el ramo de ventas, corresponda al perfil Del gerente descartable. En Argentina, este percentage va a 71\% y en Chile se estima que Sean 73\%.

Estos porcentages son de hecho altos y recuerdan que en buena parte el modelo de contratación y de producción en servicios, especialmente bacários, proviene de multinacionales, con eje de intereses anglo - americanos. El sistema de extracción de resultados es realizado sobre mano de obra calificada local, que gana enquanto actua en inflexibles acuerdos de productividad y pierde al menor deslize próprio o de inesperadas vicisitudes del mercado.

\section{LA EMPRESA MODERNA EN FOCO}

En el mundo globalizado, con la abertura de las fronteras económicas, caen lãs barreras comerciales, de precios y de transporte de mercancias.

Queda fácil al empleador de mano de obra contratar gente en un ambiente de mobilidad cresciente. De esta menera, el poder de bargaña de las empresas aumenta y el de los trabajadores individualmente disminuye. En que pesen los sindicatos, ellos son útiles enquanto el trabajador esté dentro de ellos, sindicalizados. Una vez fuera, el trabajador no es defendido y es dependiente de sus próprios contactos y capacitaciones.

La empresa moderna busca en este ambiente la obtención de resultados econômicos y usa métodos diferenciados de apropriación de resultados, conforme el tipo de trabajador y de local con el qual lida.

En este sentido, se puede desarrollar el siguiente conjunto de equaciones y de relaciones comportamentales, bajo la óptica del gerente descartable. Se mantiene en el plano general que, dentro de cierta faja de actuación:

\section{$L T=R T-D T=S(p i X q i)-S(q j X(c f u+c v u))=(p 1 \times q 1+p 2 X q 2+\ldots+p n X q n)-(C F+$ $(($ prm1 X qrm1) + ... + (prmj X qrm j) + T + 0e)}

Luego, se tiene RT a maximizar y DT a minimizar, en la faja de escala y competitividad de cada empresa.

Donde:

$\mathrm{LT}=$ lucros totales

$\mathrm{RT}=$ Ventas totales

DT $=$ Dispendios o gastos totales

$\mathrm{S}=$ somatória

$\mathrm{P}=$ precio 


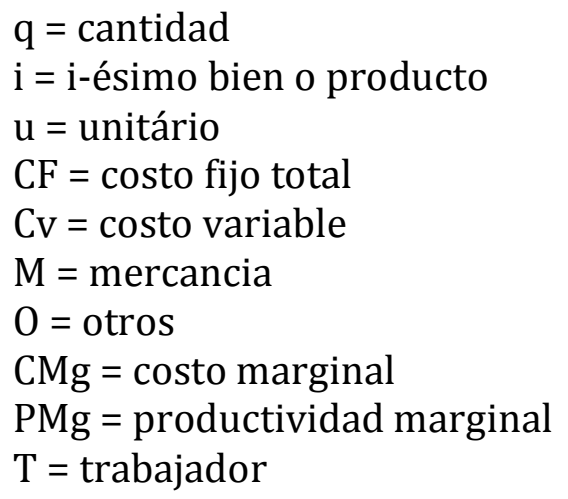

Entonces, el precio de un trabajador calificado será dado por su costo marginal, que tiene que ser menor o igual a su productividad marginal. 0 sea:

$\mathbf{p T}=\mathbf{C M g} \mathbf{T}=\mathbf{P M g T}$ y entonces

pTGD $=$ WT + DTd + BTd

Si: pT > CMgT = PmgT entonces se accepta el trabajador, porque su contratación crea margen de resultado financiero positivo al empresário, luego al contratante. Caso contrário, no se promueve la contratación.

\section{EL GERENTE EN FOCO}

El gerente de empresa tiene normalmente un conjunto de trabajos por hacer. Son clásicos y bien conocidos los que resumen sus actividades en de los teores funcionales y operacionales, así como los trabajos de comando, coordenación, concatenación, control y acompañamiento de actividades.

Contodo, son pocos y raros los estudios que analizan el tiempo de vida y de permanencia de un gerente en su puesto de trabajo. En especial, son menos conocidos aúm los periodos de tiempo de permanencia de los gerente de ventas de empresas de crédito, bancos y financieras, que en verdad rotulan esos profesionales de gerentes, quando de hecho son oficiales de crédito.

La expresión viene y deriva del inglés, donde el trabajador que concede, negocia y busca oportunidades de concesión de crédito es conocido or credit-officer.

Este tipo de gerente necesita tener un índice de productividad elevado, muy superior a la media, para permanecer en su puesto y sobrevivir en una misma empresa a largo plazo.

Esto sucede, porque las variables determinantes que afectan su productividad en El trabajo son en grande proporción externas a su control y proceso decisório.

Entre estos factores, vale establecer: quien decide si toma o no créditos, préstamos, a su conveniencia, es el cliente; la conjunctura macroeconomica afecta la demanda por créditos, con cambios provocados por la política monetária y la tasa de interés, que al subir, inibe la busca por crédito; la política fiscal reduze el ingreso neto de las empresas y acalma la demanda por préstamos destinados a la inversión; entre otros.

Por via de consequencia, el gerente de crédito tiene que obtener resultados microeconómicos y empresariales en un mundo, un ambiente, que según el momento Del ciclo de producción, 
juega contra él, independiente de su talento y capacidad.

Entonces, quando hay señales inequívocos de crisis, es probable que se mida como siendo negativa la productividad del gerente de crédito. Y a seguir, que sea dispensado. Esto es: el gerente es descartable.

Por lo tanto, hay asimetrias entre los gerentes, segun sus cargos y funciones. Enquanto los gerentes administrativos y de personal son más capazes de mantener SUS puestos de trabajo, porque sus funciones internalizan actividades y hay un menor impacto relativo de choques que vienen del exterior, sucede lo opuesto con los gerentes de ventas.

Estos, dependen de la evolución del Producto Interno Bruto - PIB, que tiene uma elevada correlación con el nivel de producción y de ventas. Quando hay una demanda agregada mayor, la demanda por créditos crece, lo que les facilita la vida profesional. Ro si ocurre lo contrário, con menor demanda agregada, con recesión, el volumen de crédito ae.

La dirección de las empresas puede leer esta situación como de reduccion de la tasa média de productividad de los gerentes y pasa a seleccionarlos, demitiendo los que parecen tener menor productividad.

\section{VANTAJAS Y DESVENTAJAS DE CONTRATAR GERENTES DESCARTABLES}

Ante la realidad anteriormente descrita, es relevante pensar y hacer reflexiones sobre quales son las ventajas y desventajas, ara una empresa competitiva, en contratar gerentes que tengan características de descartabilidad.

Los efectos de este estudio de hacen sentir en la hoja de pagos y en la combinación necessária, que se tiene que tener, entre gerentes más bien fijos y permanentes, y gerentes movivbles luego descartables.

\section{OPORTUNIDADES DE GANO Y RIESGOS EMPRESARIALES AL CONTRATAR GERENTES DESCARTABLES}

Al contratar un gerente del tipo descartable, el empresario toma una decisión racional de orden económica. Parte del presupuesto que si el trabajador es productivo y rinde, le crea valor y le dá retorno, con la cobertura simultanea de los costos variables, de manutención del trabajador.

Una vez que constate que el trabajador le ofrece resultados crecientes o al menos constantes positivos, lo mantendrá en el quadro y en la hoja de pagos. Contodo, si por un periodo definido, de, degamos, seis o más meses, el rendimiento del trabajador se prueba negativo, será demitido.

Esta última decisión es relevante, porque significa que a la amenaza de crear um "costo fijo", por plazo indeterminado, y por no saber si en el futuro los costos serán cubiertos, la empresa hace la demisión. Entonces, corta en los costos, para mantenerse equilibrada.

Más que acuerdos, lo que este modelo comportamental empresarial muestra es uma dirección vuelta a la realidad de las indicaciones dadas por los números y que es orientada a los resultados.

Esta es una manera bien competitiva de trabajar y de ejecutar el espíritu dirigista capitalista, 
en una empresa.

\section{EL MIX O LA HARMONIZACIÓN DE PERFILES: SUS VENTAJAS Y DESVENTAJAS}

Ya dice el dictado que no hay que ir ni tanto al mar, ni tanto a la tierra. 0 sea, los extremos pueden perder el sentido. El radicalismo en contratación de trabajadores em general, y de otros más específicos, como es el acso, tiene que ser estudiado y analizado con atención y precaución por los ejecutivos empresariales.

Hay ventajas y desventajas en cada modelo que se adopte, in-extremis. Por lo tanto, hace sentido buscar el equilíbrio en el proceso de selección y manutención de mano de obra especializada. Es relevante conocer ensíntesis las ventajas y desventajas de cada situación.

En el caso de contratar gente por tiempo indeterminado, las ventajas son: El trabajador se siente más seguro, luego puede produzir con más seguridad; no hay impedimiento de demitir, esta es una alternativa en caso de necesidad; el trabajador sabe que su desempenho tiene que ocurrir y ser probado.

Ya las desventajas son sobretodo las siguientes: el costo médio del trabajador sube, a lo largo del tiempo; y puede ocurrir un relajamiento de parte a parte, lo que quiere decir pérdida de eficiencia.

En el caso claro y declarado de contratar gerentes descartables, las ventajas son sobretodo económicas, puesto que los costos pueden para a qualquier momento; no hay como efecto una alta de los costos fijos; se ofrece un ambiente renovador en la empresa; quedan los más agresivos y comercialmente competentes; y la velocidad de los negócios aumenta.

En el reino de las desventajas se cuentan las siguientes: por falta de fidelidad, los más competentes trabajan como mercenários y a la mejor propuesta siguiente, quieren ir, abandonan la empresa, a menos que existan contratos que eliminan esta posibilidad; no se hace un trabajo de equipo, pues muchos gerentes luchan y concurren unos con los otros; los que salen, se sienten perdedores y pueden nutrir sentimientos contrários a los intereses de La empresa; en ciertos casos, la venganza del demitido sucede más adelante, en el mundo de los negócios.

Estos elementos muestran que el pendor, la tendencia, en una o otra dirección, tiene sus consequencias. Hay que medirlas, para saber lo que efectivamente va a dar ganos de largo plazo a la empresa.

Por las evidencias, la falta de compromiso mata y ahoga. Y el exceso de indeterminación mueve la productividad para bajo. Entonces, hace sentido detener uma parte de la mano de obra más eficiente como una ancla; con la rotatividad de un grupo algo menor, lo quanto es una situación caso a caso, que sea realmente descartable, pero altamente resultado orientado.

\section{CONSEQUENCIAS DEL EMPLEO DE GERENTES DESCARTABLES}

Al proceso de contratar, mantener o liberar mano de obra descartable, analizado em las secciones anteriores, se puede resumir con un quadro, o esquema de alternativas empresariales de contratación, con sus diferentes efectos sobre la empresa y el trabajador.

Son várias las opciones que se pueden vislumbrar en las relaciones empresa - trabajador. Segun cada situación, las acciones y reacciones se manifestarán, creando situaciones 
El gerente descartable: un dilema entre la optimización de la productividad y la minimización del bienestar social

diferenciadas de productividad, efectividad, motivación y rendimiento en El trabajo.

El acto de trabajar claramente, sin esecular sobre el futuro del trabajador, ES especialmente importante. La inseguridad derrumba la productividad. Y al quadro establecido, claro o obscuro, una vez descubierto en su realidad, se puede contraponer El mapa de reacciones del trabajador descartable. Eso lo hacemos en el quadro que sigue.

Que se note que esta dinamica tiene a ver con la Teoria de Los Juegos, de Von Neuman Morgenstern. Existen hipótesis y conforme las suposiciones, los actores hacen sus juegos. Son estos juegos del tipo: "si yo supongo que soy mantenido, y soy productivo, más que los otros, entonces permanezco por más tiempo en la empresa. Gano y puedo subir en la jerarquia”.

Quadro 1: El acto de contratar gerentes descartables: consequencias y reacciones posibles Proceso perceptual bajo quatro situaciones distintas

\begin{tabular}{|c|c|}
\hline Número & 1- La empresa contratante confiesa y define la corta temporalidad com descarte \\
\hline 1 & El contratado sabe lo que esperan de él y trabaja claramente por el tiempo definido. \\
\hline 2 & Em la medida em que el tiempo pasa, la fidelidad y la productividad caen. \\
\hline 3 & El contratado se esmera, para probar que vale, em la esperarza de ser recontratado. \\
\hline 4 & El contratado manifiesta decepción, depresión desde el comienzo, lo que acelera su descarte. \\
\hline 5 & La confesión ética de la empresa se concatena com la acción ética del contratado. \\
\hline & II - La empresa contratante no confiesa el descarte, pero lo ejecuta. \\
\hline 1 & El contratado sabe lo que esperan de él, se motiva y trabaja. Es demitido lo que le crea um choque. \\
\hline 2 & Alto o bajo, el rendimiento no define la permanencia. El demitido sufre y tiene ódio, rabia. \\
\hline 3 & Al sentirse aprovechado sin respuesta para la competencia, el demitido reage juridicamente. \\
\hline 4 & El contratado, deprimido, com rabia, seva desiludido y herido, com amor próprio bajo. \\
\hline 5 & La falta de ética empresarial puede llevar a comportamientos no éticos del funcionário. \\
\hline & III- El gerente cree en el trabajo permanente y promoción por talento. La empresa tambien. \\
\hline 1 & Empresa y colaborador hacen uma apuesta y pacto de acción permanente, com mútuo respecto. \\
\hline 2 & La productividad individual crece. \\
\hline 3 & La búsqueday conquista por clientes aumenta, por efecto motivacional. Hay uma alianza mútua. \\
\hline 4 & Caso ocurra suceso, ambos ganan y pueden ccurrir promociones. \\
\hline 5 & El costo médio de manutención de gerentes sube. La establidad tiene su costo y ganos. \\
\hline & IV - El gerente sabe del trabajo temporário y de descarte súbito. Hay promoción \\
\hline & potencial por comprobación de talento y méritos. \\
\hline 1 & La honestidad de propósitos molda esta situación. Se sabe mútuamente lo que esperar. \\
\hline 2 & Enquanto las conquistas valgan la pena, el gerente se esfuerza, la empresa lo apoya. \\
\hline 3 & Quando la situacion se invierte, ambos prueban arreglarla. No es cierto si dá resultados. \\
\hline 4 & Quando el quadro resulta endificuldades, hay tendencia em ayudarse mútuamente, com abertura. \\
\hline 5 & Al partir, después de um tiempo, los dos lados sabian de la separación, sin remorsos. \\
\hline
\end{tabular}

\section{CONCLUSIONES ESTRATÉGICAS}

Este estudio muestra que contratar, mantener y concentrar fuerzas de contratación sobre la mano de obra descartable es sobretodo arriegado.

La empresa se mantiene en las manos de un tipo de trabajador que posuye baja fidelidad con 
ella; busca oportunidades mejores y de estabilización en otros parajes; y está dispuesta a abandonar su puesto por mejores pagos en otras localidades.

Esto significa que una alta contratación percentual de trabajadores descartables ES un reductor de productividad y de estabilidad de largo plazo en las empresas.

Hace más sentido balancear y obtener perfiles de mano de obra más variado, que se puedan tambien mantener, porque equivalen a anclas en las empresas y las mantienen fijas a lo largo del tiempo.

\section{BIBLIOGRAFIA}

Academia; Revista Latinoamericana de Administración; Publicaciones del Consejo Latinoamericano de Escuelas de Administración; Facultad de Administración de La Universidad de los Andes; Bogotá; D. C. Colombia; número 1 em diante; www.administracion.uniandes.edu.co ; 2.008.

Administração de Empresas; Enciclopédia de Direção, Produção, Finanças e Marketing; Nova Cultural; Editora Abril S A; 1.986.

Distribuição; Revista de negócios dos atacadistas distribuidores; ano I em diante; 1.992 em diante; número 1 em diante; ABAD; EBC - Editora Brasileira do Comércio; www.revistadistribuição.com.br ; 2.008 .

Encontro; año I en delante; 2.001 em diante; número 1 em diante; ISSN 1679 - 0146; www.revistaencontro.com.br; 2.008.

Exame; ano 1 en delante; 1.967 em diante; números 1 em diante; Editora Abril; www.exame.com.br; 2.008.

Época; Editora Globo; número 254 en delante; Editora GLOBO; São Paulo; SP; www.epoca.com.br;

Kasznar, Istvan; El Proceso de Admisión de empleados en el Banco Francês e Brasileiro:

Ventajas y Desventajas; mimeo; Edición BFB; año 1984.

Kasznar Istvan; Comment Optimizer la Main d'Oeuvre qui Travaille en Finances et Operations de Crédit Bancaire; Études de Haute Administration; série blanche; BFB, avec recours aux données du Crédit Lyonnais - Paris, France; Banco Francês e Brasileiro año 1985.

Líderes; anos I em diante; números 1 en delante; Instituto Fórum de Líderes empresariais; www.lideres.org.br; 2.008.

Newsweek; volumes I - em delante; ISSN 0028 - 9604; nwsub@newswee.com; newsweek.msnbc.com; Estados Unidos da América; 2.008.

Revista de Administração Pública - RAP; ISSN - 0034-7612; EBAPE - Escola Brasileira de Administração Pública e de Empresas; FGV - Fundação Getúlio Vargas; 1.967 em diante; www.ebape.fgv.br; 2.008.

Revista de la Real Academia de Ciências Exactas, Físicas y Naturales; Serie A: Matemáticas; 
El gerente descartable: un dilema entre la optimización de la productividad y la minimización del bienestar social

RAC SAM números 1 a 102; ISSN 1578-7303; Religraf; Madrid; España.

Scientific American; ISSN - 0036-8733; anos 1.995 em diante; New York; NY; EUA; www.sciam.com ;2.008. 